\begin{tabular}{|c|l|}
\hline Title & $\begin{array}{l}\text { Experimental Verification of a Critical Condition for the Formation of As Cast Coarse Columnar A ustenite Grain } \\
\text { Structure in a Hyperperitectic Carbon Steel }\end{array}$ \\
\hline Author(s) & Ohno, Munekazu; Maruy ama, Masato; Matsuura, Kiyotaka \\
\hline Citation & $\begin{array}{l}\text { Metall lurgical and materials transactions A-physical metall lurgy and materials science, 46A (11), 5240-5247 } \\
\text { https:/doi.org/10.1007/311661-015-3115-x }\end{array}$ \\
\hline Issue Date & 2015-11 \\
\hline Doc URL & http://hdl.handle.net/2115/63399 \\
\hline Rights & The final publication is available at Springer via http://dx.doi.org/10.1007/s11661-015-3115-x \\
\hline Type & article (author version) \\
\hline File Information & Ohno-MMTA 46(11).pdf \\
\hline
\end{tabular}

Instructions for use 


\title{
Experimental verification of a critical condition for the formation of as-cast coarse columnar austenite grain structure in a hyperperitectic carbon steel
}

\author{
Munekazu Ohno, Masato Maruyama, Kiyotaka Matsuura
}

Corresponding author: Munekazu Ohno

Associate Professor

Division of Materials Science and Engineering, Faculty of Engineering, Hokkaido University, Kita 13 Nishi 8, Kita-ku, Sapporo, Hokkaido 060-8628, JAPAN

\begin{abstract}
Experimental verification of a critical condition for the formation of coarse columnar $\gamma$ grain (CCG) structure in as-cast hyperperitectic carbon steels, which was put forward based on theories of grain growth and phase-field simulations in early studies, is carried out by means of a Bridgman-type directional solidification experiment. The occurrence of the discontinuous and continuous grain growth processes and the resulting formation of CCG and equiaxed $\gamma$ grain structures, respectively, are demonstrated. Importantly, these changes of the as-cast microstructures and the grain growth modes are in excellent agreement with the previously-proposed critical condition of the CCG formation.
\end{abstract}




\section{INTRODUCTION}

Control of the as-cast austenite $(\gamma)$ structure in casting processes of peritectic solidified carbon steels is of great importance because the size and morphology of the $\gamma$ grain structure determine the quality of the as-cast steels ${ }^{[1-4]}$. The peritectic solidified carbon steels especially having a composition around the peritectic point have a strong tendency to undergo the formation of coarse columnar $\gamma$ grain (hereafter abbreviated as CCG) structure in continuous casting processes. The CCG structure causes the deterioration of the mechanical properties of the cast and also the occurrence of the surface cracking which is one of the serious problems reducing the yield ratio of continuously-cast slabs. Therefore, elucidation of the formation mechanism of CCG structure and also prevention of formation of CCG structure have been one of the longstanding and important issues in the field of the solidification and casting of steels [5-8].

Our experimental investigations on the evolution process of solidification microstructure in hyperperitectic carbon steels revealed that the formation of CCG is ascribable to a discontinuous grain growth process from fine $\gamma$ grains forming in the $\delta$ dendrite $^{[9,10]}$. Furthermore, a theoretical relationship indicating a critical condition for the formation of CCG structure was put forward based on theories of grain growth and phase-field simulations of the discontinuous grain growth ${ }^{[11,12]}$. The relationship is given by a balance between the cooling condition and the growth rate of CCG and it will serve as a guiding principle for the prevention of CCG formation. It is important to point out that the experimental verification of the theoretical relationship is indispensable for the practical applications.

Recently, the validity of the theoretical relationship for the critical condition of CCG formation was investigated by means of the casting experiments ${ }^{[13]}$. The relation between the cooling condition and the observed microstructural changes were consistent with the theoretical relationship and this fact supports its validity. However, further efforts are required to prove the validity and reliability of the theoretical relationship because of the following reasons. The cooling condition in the experiments of Ref. [13] was not steady state condition. The discussion in Ref. [13] accordingly involves uncertainties associated with the non-steady state condition as explained in the next section. The detailed examination of the validity and reliability of the theoretical relationship requires a solidification experiment with a precise control of the steady state cooling condition. Moreover, it is expected that when the cooling condition is controlled so as to avoid the CCG formation based on the theoretical relationship, the continuous grain growth will take place and the equiaxed $\gamma$ grain (EG) structure 
accordingly forms. However, this expectation has not been experimentally substantiated. In this study, we carry out a Bridgman-type directional solidification experiment of a hyperperitectic carbon steel in order to examine the validity and reliability of the theoretical relationship for the critical condition of CCG formation. One will see that the shape of as-cast $\gamma$ grains changes with the pulling velocity exactly according to the theoretical relationship. In addition, an evidence for the occurrence of the continuous grain growth and the resulting formation of the EG structure will be demonstrated. To explain the purpose of this study in more detail, the important findings in early works are briefly summarized and the remaining important issues are pointed out in the next section. Section III is devoted to the explanation of the experimental procedure. The results and discussion are given in Section IV, followed by the conclusion in Section V.

\section{DISCONTINUOUS GRAIN GROWTH AND THE CRITICAL CONDITION FOR ITS OCCURRENCE}

In order to make clear the purpose of the present study, we briefly summarize the previous important findings regarding the formation of CCG structure and point out the remaining issues that are actually addressed in this study.

The formation process of CCG structure in hyperperitectic carbon steels was investigated by means of a rapid unidirectional solidification experiment, the cooling condition of which is very similar to the one near the slab surface in the practical continuous casting processes ${ }^{[9,10]}$. Figure 1 (a) shows the schematic illustration of the rapid unidirectional solidification equipment in which $\mathrm{MgO}$ pipe on water-cooled copper mold was preheated to $1843 \mathrm{~K}\left(1570^{\circ} \mathrm{C}\right)$ (melt temperature) and it plays a role of a sidewall of the cast. The melted sample was cast into MgO pipe and it solidifies directionally from the bottom (copper mold side) toward the top. This equipment enables a rapid drop of the cast into intensely agitated iced water together with the $\mathrm{MgO}$ pipe and it thereby enables the analysis of the frozen structure of the solidifying sample. From the microstructural observation of the quenched samples, it was found that fine columnar $\gamma$ grain (FCG) always exists ahead of the CCG growing in the solidification direction as schematically shown in Fig. 1(b). The short-axis diameter of FCG was comparable with the primary dendrite arm spacing (DAS) of $\delta$ phase, while the diameter of CCG was about mm in size. The important fact in Fig. 1(b) is that the change from FCG to CCG is discontinuous and the boundary between FCG and CCG regions is clearly identified. This boundary is called FCG/CCG region boundary (FCRB) in this paper. The temperature at the FCRB was always close to the temperature for completion

of $\gamma$ transformation, $T_{\gamma}$, during the solidification. Hence, the FCG region corresponds to 
liquid $+\gamma$ two-phase field, while the CCG is in $\gamma$ single-phase field. These microstructural features were observed in an earlier study using a Bridgman-type directional solidification experiment ${ }^{[14]}$. These microstructural features indicate that the formation of CCG is ascribable to a discontinuous grain growth mechanism ${ }^{\text {[9] }}$ as explained below.

In the beginning of the solidification, the columnar dendrites of $\delta$ phase develop in the direction of temperature gradient from the mold wall. The $\gamma$ phase appears near the mold side and grows to CCG, although this process was not elucidated in detail. Ahead of the CCG, new $\gamma$ grains form in the $\delta$ dendrite below the peritectic reaction temperature $T_{\mathrm{p}}$ and they grow to FCG. In the FCG region, the liquid phase surrounding the FCGs exerts the pinning force on the grain boundary of FCGs in the short-axis direction. Hence, the short-axis diameter of FCG becomes comparable with the primary DAS of the $\delta$ dendrites. The liquid phase in the FCG region also retards the motion of FCRB, viz. the growth of CCG in the direction of temperature gradient. After the liquid disappears and thereby the pinning force vanishes below $T_{\gamma}$, the CCGs grow in the direction of temperature gradient at the expense of FCGs. This growth process is classified into a discontinuous grain growth process and it is characterized by the motion of FCRB in the direction of temperature gradient.

The occurrence of such discontinuous grain growth in the as-cast $\gamma$ grain structures was confirmed by the phase field simulation of the grain growth from the FCG structure ${ }^{[11,12]}$. Moreover, the critical condition for the discontinuous growth to start and continue was examined in detail. It is given by ${ }^{[11]}$,

$$
V_{T} \leq V_{C C G},
$$

where $V_{\mathrm{T}}$ is the migration velocity of the position at which the temperature reaches $T_{\gamma}$ and this velocity is called the thermal velocity. $V_{\text {CCG }}$ is the migration velocity of FCRB and, based on the theories of grain growth ${ }^{[15,16]}$, the following relation is obtained ${ }^{[11]}$,

$$
V_{C C G}=M\left(T_{\gamma}^{*}\right) \frac{1}{\xi \cdot d_{F C G}} .
$$

Here, $M\left(T_{\gamma}{ }^{*}\right)$ is the kinetic constant for $\gamma$ grain growth at a temperature $T_{\gamma}{ }^{*}=T_{\gamma}-$ $G \cdot \xi \cdot d_{\mathrm{FCG}}$ with a temperature gradient $G$. $d_{\mathrm{FCG}}$ is the short-axis diameter of FCG and it corresponds to the primary DAS of $\delta$ phase as described above. $\xi$ is a constant determined by the local shape of FCRB. During the formation process of CCG, the migration of FCRB in the direction of temperature gradient is inhibited by the pinning effect of liquid phase in FCG region ${ }^{[9]}$. As the cooling proceeds, the liquid phase disappears and the pinning effect accordingly vanishes in the FCG region. Then, the 
FCRB can move and the discontinuous grain growth thereby proceeds. However, the temperature in the FCG region is always higher than that in the CCG region due to the temperature gradient and therefore the grain boundary mobility in the FCG region is generally higher that in the CCG region. Hence, if the liquid phase disappears quickly, in other words, the position of $T_{\gamma}$ moves at a velocity higher than the migration velocity of FCRB ( $V_{\mathrm{T}}>V_{\mathrm{CCG}}$ ), the continuous grain growth of FCGs takes place instead of the discontinuous grain growth. In this case, the EG structure will form. The discontinuous grain growth is dominant when $V_{\mathrm{T}}$ is lower than $V_{\mathrm{CCG}}$ and this condition is exactly represented by Eq. (1). The validity of Eq. (1) was supported by the phase-field simulations ${ }^{[11]}$.

The validity of Eq. (1) was examined by means of casting experiments using the different types of molds (metallic and ceramic molds) in a recent study ${ }^{[12]}$. The as-cast $\gamma$ grain structures in all the samples consist of the CCG and EG regions. The position where the microstructure changes from CCG to EG, which is called transition depth according to Ref. [13], depended on the type of the molds, viz., the cooling condition. Since the cooling condition was not steady state condition in these casting experiments, $V_{\mathrm{T}}$ and $V_{\mathrm{CCG}}$ depended on the position of the samples. In addition, both the primary DAS (viz., $d_{\mathrm{FCG}}$ ) and the numerically estimated $V_{\mathrm{T}}$ at the transition depth were different for the different cooling conditions. However, the values of $M\left(T_{\gamma}{ }^{*}\right)$ estimated by substituting $d_{\mathrm{FCG}}$ and $V_{\mathrm{T}}$ at the transition depth into Eqs. (1) and (2) were almost identical in all the casting experiments. That is, almost a unique value of $M\left(T_{\gamma}{ }^{*}\right)$ was obtained even though the critical values of $V_{\mathrm{T}}$ and $d_{\mathrm{FCG}}$ were different depending on the casting condition. This is the important fact supporting the validity of Eq. (1). However, a care has to be paid to the following points. The discussion of Ref. [13] is based on the assumption that the relation of $V_{\mathrm{T}}=V_{\mathrm{CCG}}$ is satisfied exactly at the transition depth as long as Eq. (1) is valid. This assumption should basically be reasonable as demonstrated in our phase-field simulations of the discontinuous grain growth under the non-steady state cooling condition (see Fig. 7 of Ref. [11]). However, from such phase-field simulations one can also understand that there is some variation in the transition depth and the transition depth does not exactly coincide with the position of $V_{\mathrm{T}}=V_{\mathrm{CCG}}$. In fact, a relatively large error associated with the variation of the transition depth was considered in the estimation of $M\left(T_{\gamma}{ }^{*}\right)$ in Ref. [13], which impairs the accuracy of the validation. Hence, the solidification experiment under steady state cooling condition is indispensable for further detailed examination of the validity and reliability of Eq. (1). In addition, the direct evidence has not been demonstrated regarding the occurrence of the continuous grain growth and the resulting formation of EG structure when $V_{\mathrm{T}}>$ 
$V_{\text {CCG. }}$ These points are addressed in this study by means of the Bridgman-type directional solidification experiment.

\section{EXPERIMENTAL PROCEDURES}

We employed a 0.2 mass\% carbon steel rod, the chemical composition of which is $0.204 \% \mathrm{C}, 0.208 \% \mathrm{Si}, 0.805 \% \mathrm{Mn}, 0.015 \% \mathrm{P}, 0.004 \% \mathrm{~S}, 0.041 \% \mathrm{Al}$ and $0.0064 \% \mathrm{~N}$ (in mass\%). This is one of typical carbon steels which have a strong tendency to exhibit the CCG structure in casting processes ${ }^{[3,5]}$. The equilibrium liquidus, $T_{\mathrm{L}}$, the peritectic reaction temperature $T_{\mathrm{p}}$ and $T_{\gamma}$ of the steel are 1788 (1515), $1762(1489)$ and $1749 \mathrm{~K}\left(1476^{\circ} \mathrm{C}\right)$ according to the thermodynamic calculation ${ }^{[17]}$. This sample was directionally solidified in the Bridgman-type vertical tubular furnace. The sample setup inside the furnace is schematically shown in Fig. 2(a). The sample of 150 g having a cylindrical shape with a center hole of $7 \mathrm{~mm}$ diameter was put into an $\mathrm{Al}_{2} \mathrm{O}_{3}$ crucible with an inner diameter of $20 \mathrm{~mm}$ and a depth of $90 \mathrm{~mm}$. The $\mathrm{Al}_{2} \mathrm{O}_{3}$ suspension tube with an outer diameter of $6 \mathrm{~mm}$ was inserted into the sample through the hole. The B-type thermocouples were inserted into the sample through the $\mathrm{Al}_{2} \mathrm{O}_{3}$ suspension tube to measure the temperatures inside the sample. Then, the top of the crucible was closed by the ceramic cap having two holes; one is for the $\mathrm{Al}_{2} \mathrm{O}_{3}$ suspension tube and the other is for a small $\mathrm{Al}_{2} \mathrm{O}_{3}$ tube with a diameter of $2 \mathrm{~mm}$ which was used for the ventilation. The crucible, cap and the $\mathrm{Al}_{2} \mathrm{O}_{3}$ suspension tubes were fixed by $\mathrm{Al}_{2} \mathrm{O}_{3}$ cement. The Bridgman-type directional solidification equipment used in this study is schematically shown in Fig. 2(b). It consists of the vertical furnace core pipe with an inner diameter of $60 \mathrm{~mm}$ and a length of $1000 \mathrm{~mm}, \mathrm{MoSi}_{2}$ heating elements and lifting/lowering device outside the furnace. The crucible with the sample shown in Fig. 2(a) was put inside the vertical furnace core pipe and it was suspended from above by fixing the $\mathrm{Al}_{2} \mathrm{O}_{3}$ suspension tube to the lifting/lowing device outside the furnace. The furnace core pipe was hermetically-closed with the upper and lower caps of water-cooled steels. The lower cap is manually openable by sliding the plate. The furnace core pipe was evacuated first and then filled with Ar of 99.9995\% purity. The Ar flow was kept all through running of the furnace.

The temperature at the center height of the vertical furnace pipe is the highest and it decreases as the height (position) decreases. The sample was first placed near the center height of the vertical furnace where the temperature is about $1823 \mathrm{~K}\left(1550^{\circ} \mathrm{C}\right)$. The sample was melted at about $1823 \mathrm{~K}\left(1550^{\circ} \mathrm{C}\right)$ inside the furnace and held for 30 min.. Then, it was moved downward at the prescribed descent velocity $V$. The sample was thereby solidified upward from the bottom. We focused on four descent velocities, 
$V=0.05,0.10,0.20$ and $0.43 \mathrm{~mm} / \mathrm{s}$. While the temperature profile in the vertical direction inside the furnace was kept constant with time during the solidification process, the temperature gradient gradually increases from about $1 \mathrm{~K} / \mathrm{mm}$ to $5 \mathrm{~K} / \mathrm{mm}$ with decrease in the position from the center to the lower position in the furnace. Although not demonstrated here,, it was found in the present experiments that the cooling process largely deviates from the steady state condition until the sample is solidified up to about $15 \mathrm{~mm}$ height. We accordingly focused on the microstructure in a region above $15 \mathrm{~mm}$ height of the sample. The value of $V_{\mathrm{T}}$ was estimated for each descent velocity by measuring the temperatures inside the sample at 20 and $30 \mathrm{~mm}$ heights. It was carried out by measuring the timings at which the temperature reaches $T_{\gamma}$ in the cooling curves at 20 and $30 \mathrm{~mm}$ heights. If an error of $\pm 2 \mathrm{~mm}$ in the measurement of the distance between the thermocouple is assumed, the estimated error of $V_{\mathrm{T}}$ is about $10 \%$ in all the cases. Table 1 shows the measured values of the cooling rate $\dot{T}$, the temperature gradient $G$ and the thermal velocity $V_{\mathrm{T}}$ for each descent velocity. $\dot{T}$ is the value near $T_{\gamma}$ and $G$ was obtained from the relation $G=\dot{T} / V_{T}$. It is noted in Table 1 that the cooling rate first increases and then decreases as the descent velocity increases. On the other hand, it was found that the cooling rate during $\delta$ solidification (1788-1762 $\mathrm{K})\left(1515-1489^{\circ} \mathrm{C}\right)$ increases with increase in $V$ monotonically as is the case of the usual directional solidification experiments. More specifically, the cooling rates during $\delta$ solidification were measured to be $0.04 \mathrm{~K} / \mathrm{s}$ for $V=0.05 \mathrm{~mm} / \mathrm{s}, 0.14 \mathrm{~K} / \mathrm{s}$ for $0.1 \mathrm{~mm} / \mathrm{s}$, $0.17 \mathrm{~K} / \mathrm{s}$ for $0.2 \mathrm{~mm} / \mathrm{s}$ and $0.2 \mathrm{~K} / \mathrm{s}$ for $0.42 \mathrm{~mm} / \mathrm{s}$. The cooling rates near $T_{\gamma}$ shown in Table 1 should be more sensitive to the temperature distribution inside the furnace and the heat extraction from the bottom of the crucible. In the cases of $V=0.05,0.1$ and 0.2 $\mathrm{mm} / \mathrm{s}$, the temperatures inside the sample should change almost in accordance with the temperature distribution inside the furnace, which can be understood from the fact that the values of $V_{\mathrm{T}}$ are almost the same as the values of $V$ in this range. However, in the case of $V=0.42 \mathrm{~mm} / \mathrm{s}, V_{\mathrm{T}}$ was measured to be $0.34 \mathrm{~mm} / \mathrm{s}$ which is lower than $V$. It means that the temperature distribution inside the sample deviated from the temperature distribution inside the furnace due to the high descent velocity of $0.42 \mathrm{~mm} / \mathrm{s}$. However, it should be emphasized that our main concern is neither the cooling rate nor temperature gradient. According to the critical condition given by Eq. (1), it is $V_{\mathrm{T}}$ that determines the occurrence of the formation of CCG. We henceforth use $V_{\mathrm{T}}$ instead of $V$ in the discussion.

The samples were completely solidified inside the furnace. The height of the solidified sample was about $60 \mathrm{~mm}$. It was vertically sectioned, polished and etched 
with 3\% nital and Oberhoffer's solutions to observe the as-cast $\gamma$ grain and dendrite structures, respectively. In addition, in order to investigate the microstructural evolution during the solidification, some of the solidifying samples were quenched by dropping them into strongly-agitated iced water after opening the lower cap of the directional solidification furnace.

\section{RESULTS AND DISCUSSION}

Figure 3 shows the as-cast $\gamma$ grain structures in the samples solidified at different $V_{\mathrm{T}}$. Since some of the grain boundaries are not clearly visible in these printed images, the grain structure traced from the macrograph is shown on the right side of each micrograph. As mentioned in the previous section, we shall focus on the microstructure above about $15 \mathrm{~mm}$ height from the bottom. When $V_{\mathrm{T}}$ is $0.075 \mathrm{~mm} / \mathrm{s}$, the CCGs form in the whole observation area (above $15 \mathrm{~mm}$ height). The structure changes gradually with increase in $V_{\mathrm{T}}$. To be more specific, the columnar grains become relatively fine and the number of EGs increases with increase in $V_{\mathrm{T}}$. The important finding is that the as-cast $\gamma$ grain structure entirely changes to relatively fine EG structure by increasing $V_{\mathrm{T}}$ up to $0.34 \mathrm{~mm} / \mathrm{s}$. The microstructural change shown in Fig. 3 demonstrates that it is possible to completely avoid the formation of CCG by controlling $V_{\mathrm{T}}$.

In order to clarify the mechanism of the microstructural change observed in Fig. 3 , we investigated the microstructures during the solidification in the quenched samples. Figure 4 shows the optical micrographs for (a) dendrite and (b) $\gamma$ grain structures in the sample solidified at $V_{\mathrm{T}}=0.0075 \mathrm{~mm} / \mathrm{s}$ and quenched into iced water before the completion of the solidification. The columnar dendrites develop upward from the lower part of the sample. On the other hand, three regions are clearly distinguishable in the $\gamma$ grain structure. Very fine grains and CCGs form in the upper and lower parts, respectively, and FCGs form between them. The structure discontinuously changes from FCG to CCG and the FCRB can be thereby identified. As reported in Refs. [9, 14], the temperature at the FCRB corresponds to $T_{\gamma}$. Hence, the CCG region is in $\gamma$ single-phase field, while the FCG region corresponds to liquid $+\gamma$ two-phase field. It is noted that the height of FCG region is about $5 \mathrm{~mm}$ and the temperature at the upper edge of FCG region was very close to $T_{\mathrm{p}}$ according to the measured temperature gradient in Table 1. Hence, the region of very fine grains in Fig. 4(b) corresponds to liquid $+\delta$ two-phase field.

According to the microstructural observation, the FCG structure seems to form via nucleation of the $\gamma$ phase inside the $\delta$ dendrites. However, its formation process has 
not been elucidated in detail. The peritectic reaction might be related to the formation of FCG structure. The kinetics of peritectic reaction was investigated by means of confocal laser scanning microscope ${ }^{[18,19]}$ and we have recently investigated the kinetics of peritectic reaction at different degree of undercooling by means of phase-field simulations ${ }^{[20]}$. According to these previous investigations, the peritectic reaction velocity is quite fast and the growth front of $\gamma$ phase forming by the reaction should easily follow the solidification velocities (descent velocities) we focused in the present paper. It means that the FCG structure can easily form in the range of the descent velocities used here, provided that the FCG structure originates from the peritectic reaction process.

As described in Section II, the CCG structure shown in Fig. 3 is considered to have developed by the discontinuous grain growth mechanism which is characterized by the upward motion of FCRB in Fig. 4(b). The FCRB is always pinned by the liquid phase in FCG region and the upward motion of FCRB occurs when $V_{\mathrm{T}}$ is lower than $V_{\text {CCG. }}$ On the other hand, it is expected that when $V_{\mathrm{T}}>V_{\mathrm{CCG}}$ is satisfied, the discontinuous grain growth becomes non-dominant and the continuous grain growth partly or entirely start, resulting in the formation of EGs. Shown in Fig. 5 are the microstructures in the sample solidified at $V_{\mathrm{T}}=0.34 \mathrm{~mm} / \mathrm{s}$ and quenched into iced water before the completion of the solidification. The columnar dendrites develop upward from the lower part of the sample as is similar to Fig. 4(a). However, the $\gamma$ grain structure in Fig. 5(b) is quite different from Fig. 4(b). Except for the bottom region of Fig. 5(b), the grains are very fine and relatively coarse in the upper and lower regions, respectively. The transition position of the grain structure is not clearly identified in contrast to Fig. 4(b). According to the temperature measurement, the position of $T_{\gamma}$ is located at the middle of this micrograph. Hence, there exist liquid $+\delta$ and liquid $+\gamma$ two-phase fields in the upper half of this micrograph. However, the clear distinction between these two-phase fields is difficult. This should be because under this high $V_{\mathrm{T}}$ condition, the $\gamma$ phase in liquid $+\gamma$ two-phase region was not allowed to grow to be large during the solidification, which makes it difficult to distinguish between the $\gamma$ grains in liquid $+\gamma$ two-phase region and those appearing in the quenching operation. When the region indicated by A in Fig. 5(b) is considered, the grains gradually become large from the upper to lower part. Hence, the continuous grain growth takes place in this region. On the other hand, in the region $\mathrm{B}$, the grain structure looks discontinuous and therefore, the discontinuous grain growth may partly occur in this sample. However such discontinuous growth is not dominant enough to allow the columnar grains to keep growing to CCGs. Hence, the grains are relatively equiaxed shape in the lower half of 
this micrograph.

As demonstrated above, the CCG structure can be avoided and alternatively the EG structure can be obtained by increasing $V_{\mathrm{T}}$. This is qualitatively consistent with the expectation based on Eq. (1). This fact indicates that the microstructural change observed in Fig. 3 is attributed to the change of the dominant mode of grain growth from the discontinuous to continuous one. Now our focus is directed at the quantitative accuracy of Eq. (1). In order to estimate $V_{\text {CCG }}$, the primary DAS of $\delta$ dendrite $\lambda_{1}$ was measured in all the samples shown in Fig. 3, since $d_{\mathrm{FCG}}$ in Eq. (2) can be approximated by $\lambda_{1}{ }^{[9]}$. The results are shown in Fig. 6 where the circle symbols represent the measured values of $\lambda_{1}$ and its vertical axis corresponds to the left side axis. $\lambda_{1}$ gradually decreases with increase in $V_{\mathrm{T}}$. However the variation of $\lambda_{1}$ is not substantial in this range of $V_{\mathrm{T}}$. By substituting these values into Eq. (2), $V_{\mathrm{CCG}}$ was estimated and the results are shown in Fig. 6 . The closed square plots with the solid line indicate $V_{\text {CCG }}$ and its vertical axis corresponds to the right side one. We used $M\left(T_{\gamma}{ }^{*}\right)=2.2 \times 10^{-8} \mathrm{~m}^{2} / \mathrm{s}$ for the carbon steel of our focus ${ }^{[21]}$ and $\xi=0.45$ which was estimated by considering the effect of liquid phase on the motion of FCRB ${ }^{[13]}$. $V_{\text {CCG }}$ does not change significantly and it approximately takes $0.1 \mathrm{~mm} / \mathrm{s}$. The dashed line in Fig. 6 is the diagonal line along which $V_{\mathrm{T}}=V_{\mathrm{CCG}}$ holds. The solid line intersects with the diagonal line at $V_{\mathrm{T}}=0.0875$ $\mathrm{mm} / \mathrm{s}$, indicating that the critical velocity is $0.0875 \mathrm{~mm} / \mathrm{s}$. Hence, the discontinuous grain growth should be dominant when $V_{\mathrm{T}}$ is lower than $0.0875 \mathrm{~mm} / \mathrm{s}$ according to Eq. (1).

Figure 7 shows the measured aspect ratio of the $\gamma$ grains in all the samples shown in Fig. 3. It decreases with increase in $V_{\mathrm{T}}$. As described above, the discontinuous grain growth should be dominant at $V_{\mathrm{T}}=0.075 \mathrm{~mm} / \mathrm{s}$ according to the microstructure in Fig. 3(a) and 4(b). The aspect ratio of $\gamma$ grains in Fig. 3(a) is as high as about 4. By slightly increasing $V_{\mathrm{T}}$ up to $0.088 \mathrm{~mm} / \mathrm{s}$, the aspect ratio suddenly drops to about 2.7, indicating that the continuous grain growth starts to takes place partly. The vertical solid line in Fig. 7 denotes the critical velocity of $0.0875 \mathrm{~mm} / \mathrm{s}$ estimated above. It is seen that the critical velocity predicted from Eq. (1) is in excellent agreement with the result of microstructural observation. This fact strongly supports the quantitative accuracy of Eq. (1). Therefore, it can be said that Eq (1) is quite reliable and it is utilized as the guiding principle for the prevention of the formation of CCG structure in peritectic solidified carbon steels.

As discussed above, the central quantity determining the occurrence of the CCG formation is $V_{\mathrm{T}}$ which is the moving velocity of the position where the temperature reaches $T_{\gamma}$, viz., the liquid phase vanishes. $V_{\mathrm{T}}$ can be varied by controlling 
the cooling condition just before the end of the solidification. On the other hand, the migration velocity of FCRB, $V_{\mathrm{CCG}}$, is determined by $d_{\mathrm{FCG}}$ which is the short-axis diameter of FCG. As already mentioned, $d_{\mathrm{FCG}}$ is generally comparable with the primary DAS of $\delta$ dendrite and $V_{\text {CCG }}$ can be accordingly varied by controlling cooling process during the $\delta$ solidification, in other words, the early stage of the solidification. Therefore, the control of the as-cast $\gamma$ grain structure in peritectic solidified carbons steels should be tackled by focusing on the cooling conditions in the early and/or late stages of the solidification process. In this regard, it is important to note that the control of $V_{\mathrm{CCG}}$ requires the knowledge of the dependence of $d_{\text {FCG }}$ or $\lambda_{1}$ on the cooling condition ${ }^{[22,23]}$. Also, it is noted that the relationship of Eq. (1) denotes the condition for the discontinuous grain growth to be dominant and it does not represent the condition for the continuous grain growth to be dominant as is understood from Fig. 7. The short-axis diameter of FCGs is not spatially uniform in the FCG structure and the discontinuous grain growth easily occurs in the region where FCGs are relatively small. Hence, the condition for the continuous grain growth to dominantly take place is determined by the size distribution of short-axis diameter of FCGs which should be closely related to the size distribution of the primary DAS. The elucidations of the size distribution of FCGs and the condition for the continuous grain growth remain as an important future work. Furthermore, we have not taken into account the elastic strain produced during $\delta / \gamma$ transformation. If the elastic strain energy contributes to the migration of FCRB, we have to consider the driving force for the migration of FCRB due to the difference in the elastic strain energy between CCG and FCG as well as the capillary force in Eq. (2). It will lead to the increase of $V_{\mathrm{CCG}}$ and thus the increase of critical velocity. This point should be investigated in detail in a future work.

\section{CONCLUSION}

The prevention of formation of coarse columnar $\gamma$ grain (CCG) structure in peritectic solidified carbon steels is one of the longstanding and important issues in the field of solidification and casting of steels. Our recent investigations revealed that the formation of the CCG structure originates from the discontinuous grain growth process from fine columnar $\gamma$ grain (FCG) structure which forms in liquid $+\gamma$ two-phase field ${ }^{[9}$, 10]. In addition, the theoretical relationship for the critical condition for the occurrence of the discontinuous grain growth given by Eq. (1) was put forward based on the theories of grain growth ${ }^{[11]}$. Although the validity of this relationship was examined by means of the casting experiments in the early study ${ }^{[13]}$, it involves uncertainties associated with the non-steady state cooling condition. Also, there has been no direct 
evidence to indicate that when the critical condition for the formation of CCG structure is avoided, the continuous grain growth takes place and the equiaxed grain (EG) structure accordingly develops. In the present study, we have carried out the Bridgman-type directional solidification experiment of a hyperperitectic carbon steel. It was demonstrated that the as-cast $\gamma$ grain structure changes from CCG to EG structure with increase in the migration velocity of $T_{\gamma}$ position, $V_{\mathrm{T}}$, and this variation of microstructure is in quantitative agreement with the theoretical relationship. In addition, the direct evidence for the occurrence of the continuous grain growth and the resulting EG formation was demonstrated. Although our focus in this paper has been directed at only hyperperitectic carbon steels, the CCG structure in hypoperitectic carbon steel also originate from the discontinuous grain growth in which the pinning phase on the migration of FCRB corresponds to $\delta$ phase ${ }^{[24]}$. Hence, the critical condition discussed in this paper should be applied to the hypoperitectic carbon steels.

\section{ACKNOWLEDGEMENTS}

This work is partly supported by The $19^{\text {th }}$ Steel making Committee's Research Grant, JSPS, Japan. 


\section{REFERENCES}

[1] L. Schmidt, Å. Josefsson: Scand. J. Metall., 1974, vol. 3, pp. 193-99.

[2] B. Mintz, J.M. Arrowsmith: Met. Technol., 1979, vol. 6, pp. 24-32.

[3] Y. Maehara, K. Yasumoto, Y. Sugitani, K. Gunji: Trans. ISIJ, 1985, vol. 25, pp. 1045-52.

[4] D.N. Crowther, B. Mintz: Mater. Sci. Tehcnol., 1986, vol. 2, pp. 951-55.

[5] K. Matsuura, Y. Itho, K. Matsubara: Tetsu-to-Hagane, 1990, vol. 76, pp. 714-21.

[6] J. Reiter, C. Bernhard, H. Presslinger: Mater. Charact., 2008, vol. 59, 737-46.

[7] N. Yoshida, Y. Kobayashi, K. Nagai, Tetsu-to-Hagané, 90 (2004), pp.198-205.

[8] Y. Kobayashi, S. Iwasaki, K. Nakazato, T. Hibaru, S. Kuroda, N. Sakuma, N. Yoshida and K. Nagai, ISIJ Int., 48 (2008), pp.344-49.

[9] S. Tsuchiya, M. Ohno, K. Matsuura and K. Isobe: Acta Mater., 2011, vol. 59, pp. 3334-42.

[10] S. Tsuchiya, M. Ohno, K. Matsuura: Acta Mater., 2012, vol. 60, pp. 2927-38.

[11] M. Ohno, S. Tsuchiya, K. Matsuura: Acta Mater., 2011, vol. 59, pp. 5700-09.

[12] M. Ohno, S. Tsuchiya, K. Matsuura: Metall. Mater. Trans. A, 2014, vol. 43A, pp. 2031-42.

[13] M. Ohno, M. Maruyama, K. Matsuura: Acta Mater., 2013,vol. 61, pp. 7334-41.

[14] T. Maruyama, K. Matsuura, M. Kudoh, Y. Itoh: Tetsu-to-Hagane, 1999, vol. 85, pp. 585-91.

[15] M. Hillert: Acta Metall., 1965, vol. 13, pp. 227-38.

[16] F.J. Humphreys: Acta Mater., 1997, vol. 45, pp. 4231-40.

[17] CompuTherm LLC: http://www.computherm.com/.

[18] H. Shibata, Y. Arai, M. Suzuki and T. Emi, Metall. Mater. Trans. B, 31B (2000), pp.981-91.

[19] D. Phelan, M. Reid and R. Dippenaar, Metall. Mater. Trans. A, 37A (2006), pp.985-94.

[20] M. Ohno and K. Matsuura, Acta Mater., 2010, vol. 58, pp. 6134-41.

[21] C. Bernhard, J. Reiter, H. Presslinger: Metall. Mater. Trans. B, 2008, vol. 39B, pp. 885-95.

[22] R. Trivedi, W. Kurz: Acta Metal. Mater., 1994, vol. 42, pp. 15-23.

[23] M. Greenwood, M. Haataja, M. Provatas: Phys. Rev. Lett., 2004, vol. 93, pp. 246101-1-4.

[24] M. Ohno, S. Tsuchiya and K. Matsuura, ISIJ Inter. (in print) . 
Table 1 The cooling rate $\dot{T}$, temperature gradient $G$ and the thermal velocity $V_{\mathrm{T}}$ measured during the directional solidification experiments at different descent velocities $V$.

\begin{tabular}{c|c|c|c}
\hline \hline$V(\mathrm{~mm} / \mathrm{s})$ & $\dot{T}(\mathrm{~K} / \mathrm{s})$ & $G(\mathrm{~K} / \mathrm{mm})$ & $V_{\mathrm{T}}(\mathrm{mm} / \mathrm{s})$ \\
\hline \hline 0.05 & 0.14 & 1.87 & 0.075 \\
\hline 0.10 & 0.42 & 4.77 & 0.088 \\
\hline 0.20 & 0.82 & 4.10 & 0.20 \\
\hline 0.42 & 0.69 & 2.03 & 0.34 \\
\hline
\end{tabular}


(a)

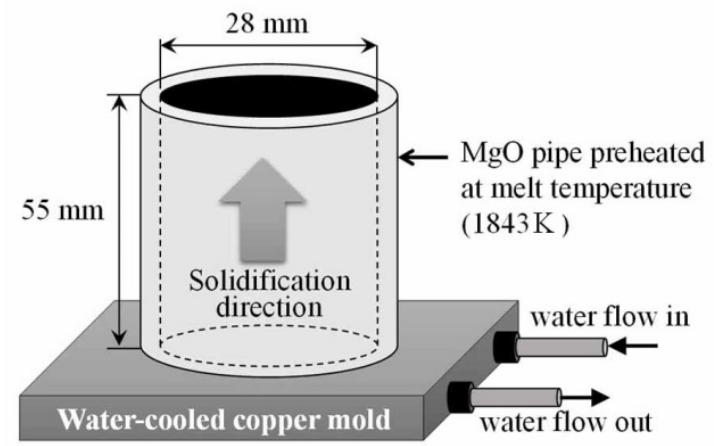

(b)

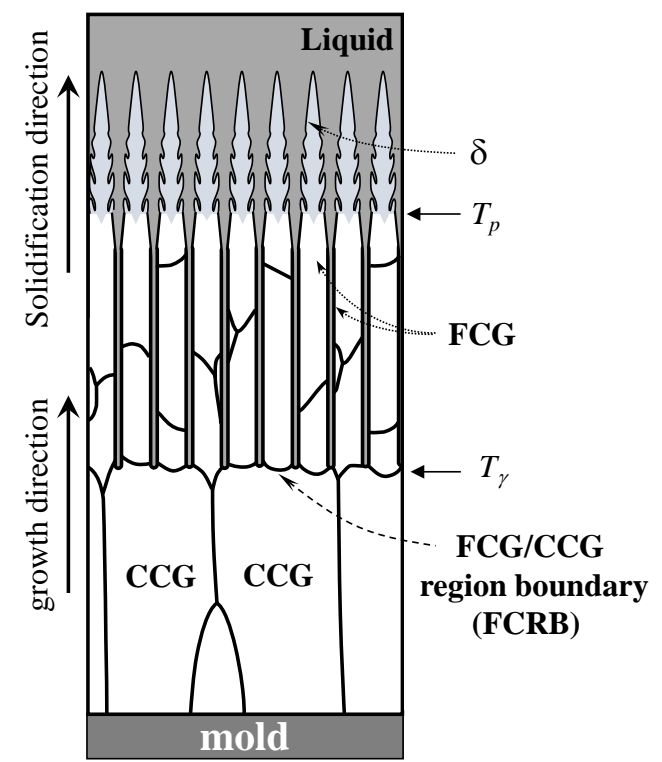

Fig. 1 Schematic illustrations of (a) rapid unidirectional solidification equipment used in the previous study ${ }^{[9,10]}$ and (b) typical microstructure during the discontinous grain growth proces observed in the quenched sample. 
(a)

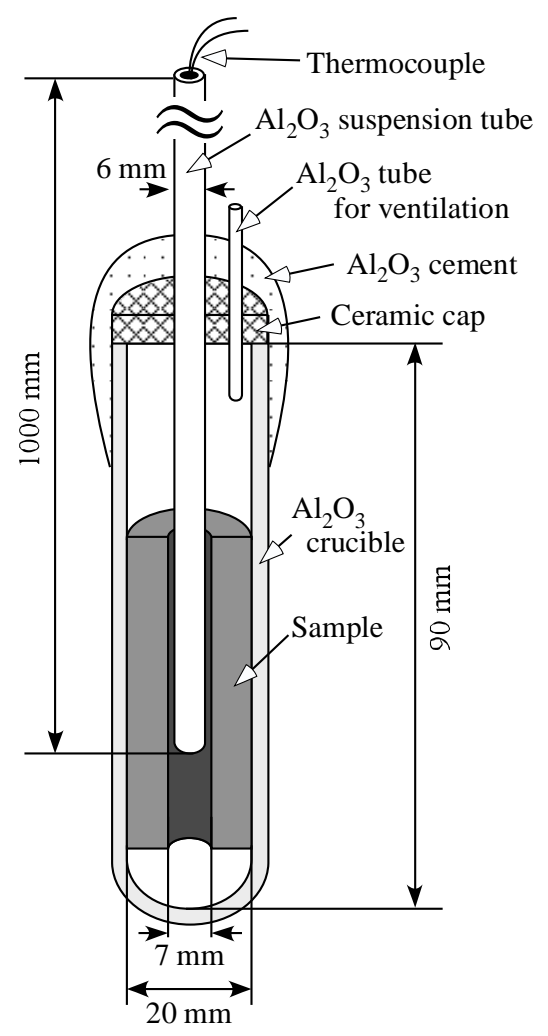

(b)

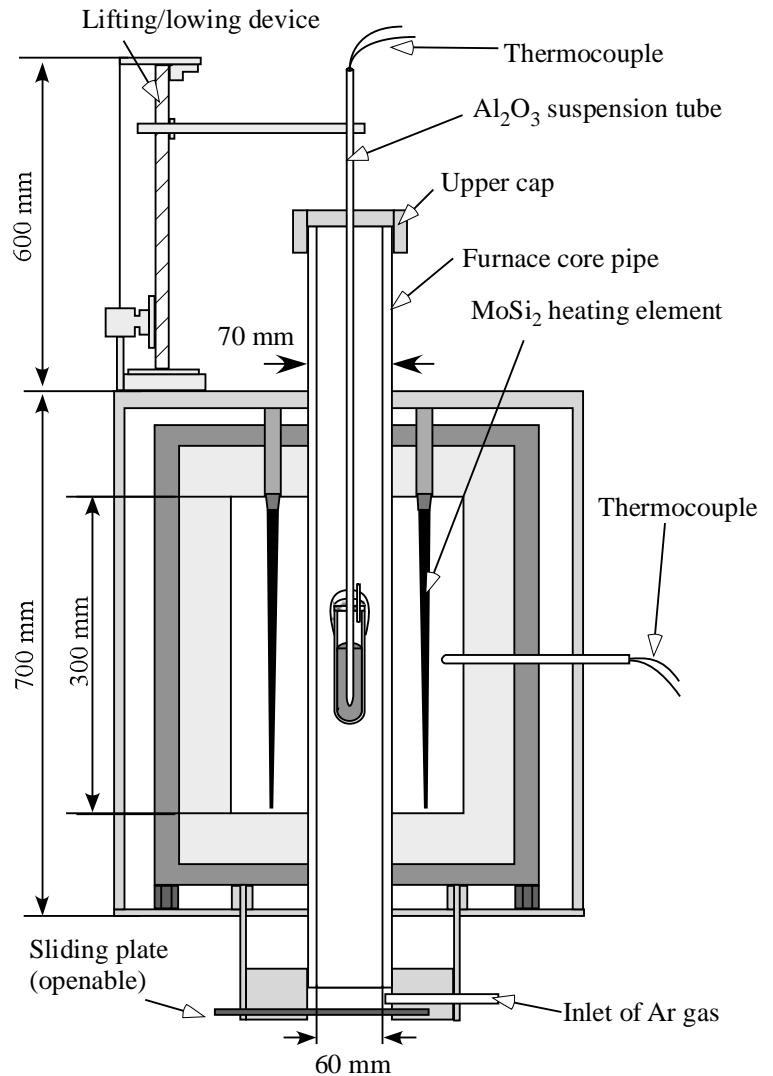

Fig. 2 Schematic illustrations of (a) sample setup inside the vertical furnace and (b) Bridgman-type directional solidification equpment used in this study. 
(a) $V_{\mathrm{T}}=0.075 \mathrm{~mm} / \mathrm{s}$
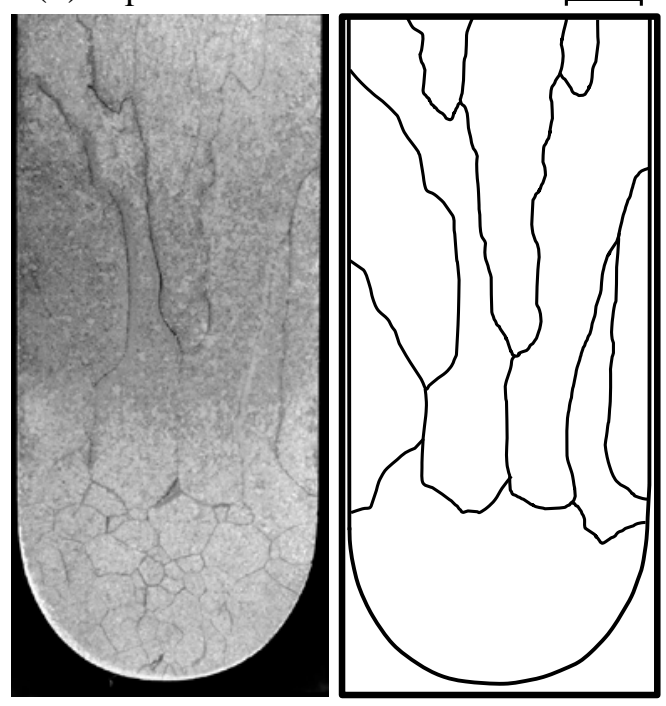

(c) $V_{\mathrm{T}}=0.20 \mathrm{~mm} / \mathrm{s}$

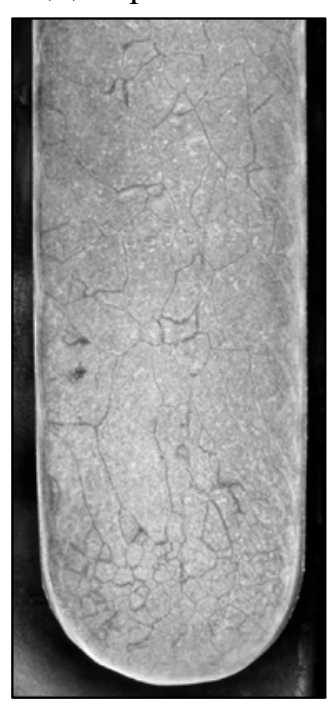

$5 \mathrm{~mm}$

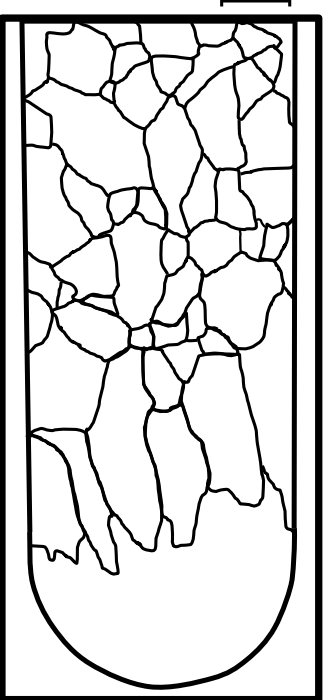

$5 \mathrm{~mm}$

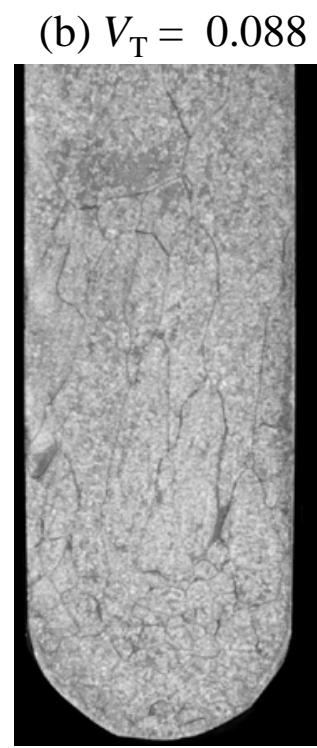

(d) $V_{\mathrm{T}}=0.34 \mathrm{~mm} / \mathrm{s}$
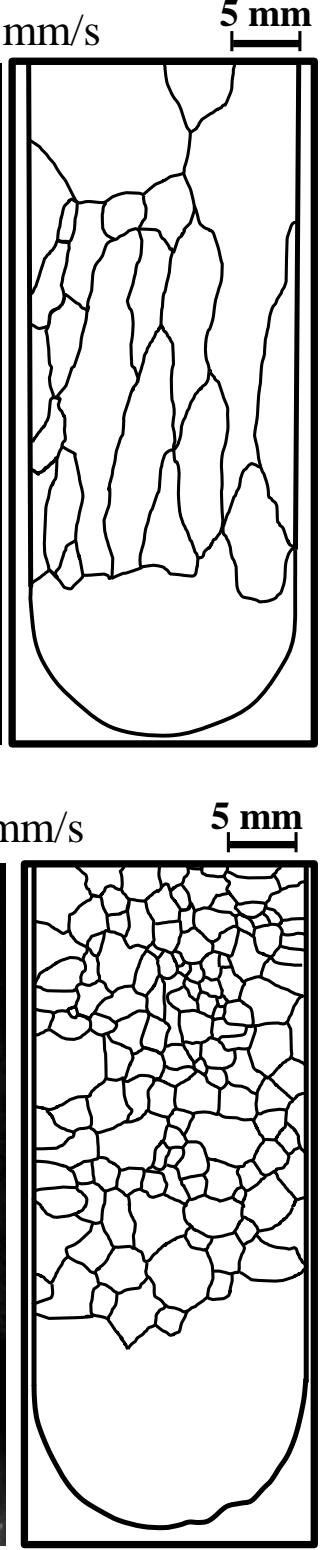

Fig. 3 As-cast $\gamma$ grain structures in 0.2 mass $\%$ steels directionally solidified at (a) $V_{\mathrm{T}}=0.075$, (b) 0.088, (c) 0.20 and (d) $0.34 \mathrm{~mm} / \mathrm{s}$. The illustration on the right side of each micrograph represents the $\gamma$ grain boundary traced from the micrograph. 
(a)

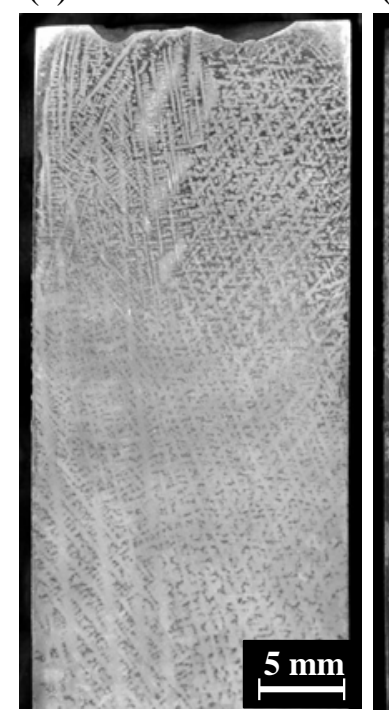

(b)

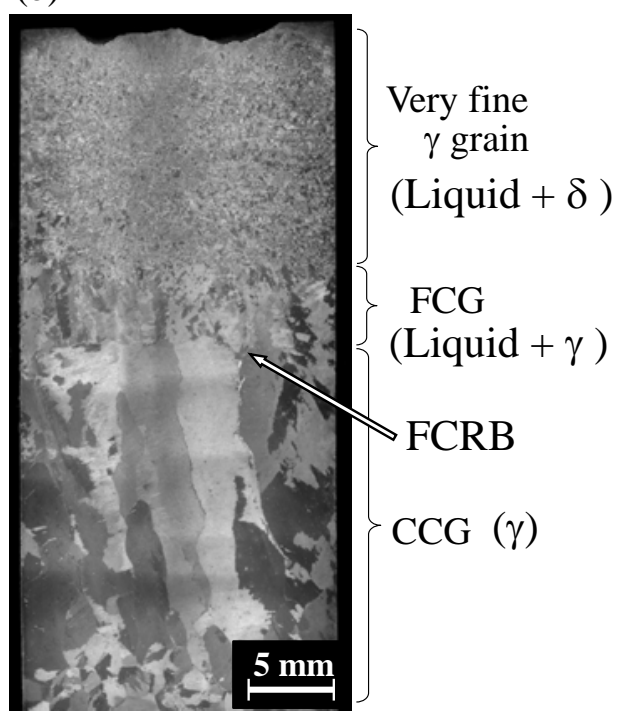

Fig. 4 Optical micrographs of (a) dendrite and (b) $\gamma$ grain structures in the sample solidified at $V_{\mathrm{T}}=$ $0.0075 \mathrm{~mm} / \mathrm{s}$ and quenched into iced water. 
(a)

(b)

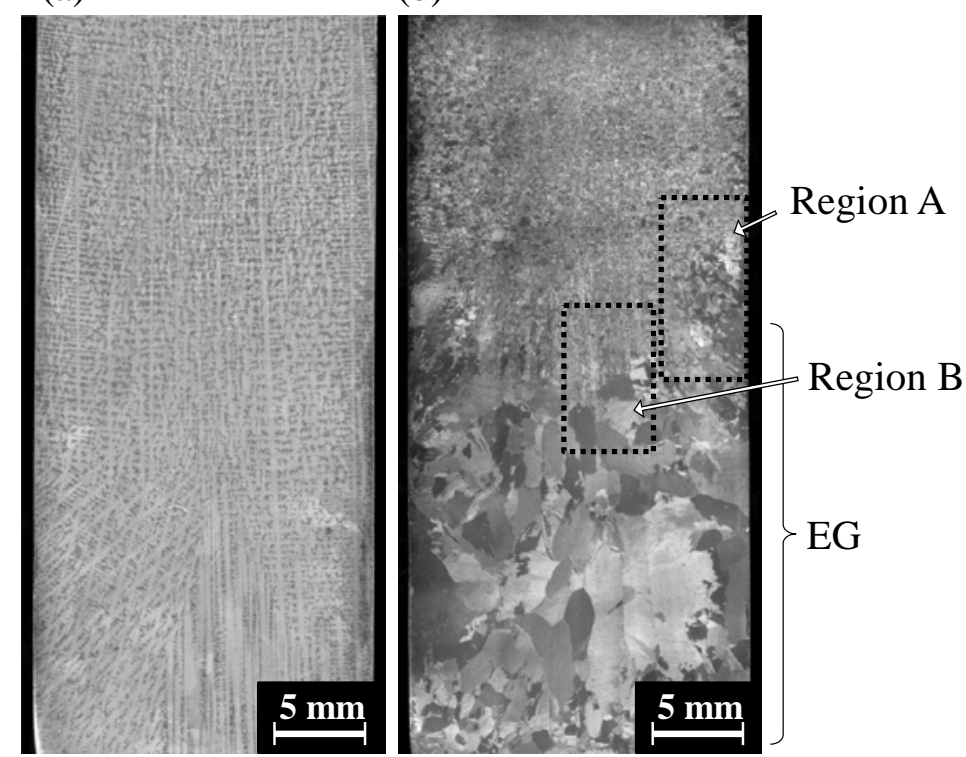

Fig. 5 Optical micrographs of (a) dendrite and (b) $\gamma$ grain structures in the sample solidified at $V_{\mathrm{T}}=$ $0.34 \mathrm{~mm} / \mathrm{s}$ and quenched into iced water. 


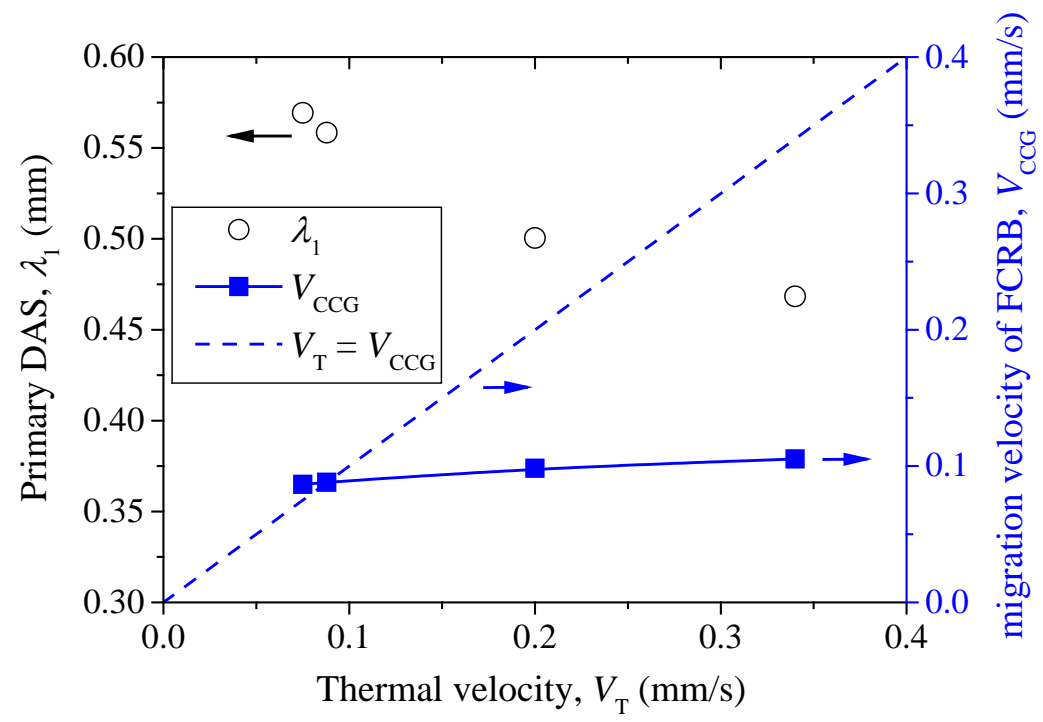

Fig. 6 The primary dendrite arm spacing (DAS), $\lambda_{1}$, (the left-hand axis) and the migration velocity of FCRB, $V_{\text {CCG, }}$ (the right-hand axis) versus the thermal velocity, $V_{\mathrm{T}}$, controlled in the directional solidification experiment 


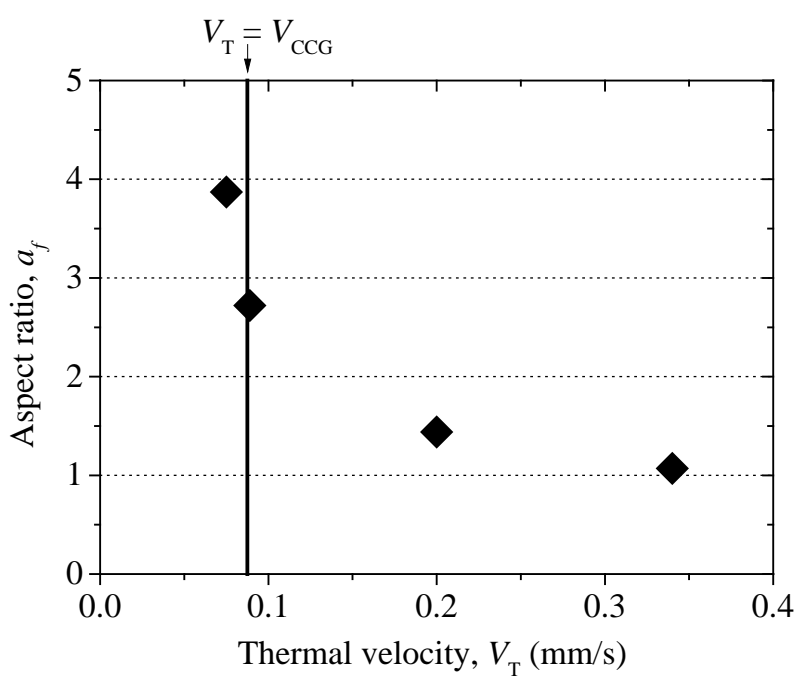

Fig. 7 The dependence of aspect ratio $a_{f}$ of as-cast $\gamma$ grains on the controlled thermal velocity $V_{\mathrm{T}}$. The vertical line indicate the critical velocity where $V_{\mathrm{T}}=V_{\mathrm{CCG}}$ holds. 